\title{
Rendimiento académico en las pruebas nacionales de matemática en colegios del área metropolitana y zonas alejadas de Costa Rica en 2013
}

\author{
Academic performance in the high school mathematics standardized test at metropolitan \\ and remote areas of Costa Rica schools in 2013
}

Mario Castillo-Sánchez

mario.castillo.sanchez@una.cr

Universidad Nacional

Heredia, Costa Rica

Jesennia-Ma. Chavarría-Vásquez

jessenia.chavarria.vasquez@una.cr

Universidad Nacional

Heredia, Costa Rica

Marcela García-Borbón

marcela.garcia.borbon@una.cr

Universidad Nacional

Heredia, Costa Rica

Recibido-Received: 2/mar/2015 / Aceptado-Accepted: 18/may/2015 / Publicado-Published: 31/ene /2016.

\begin{abstract}
Resumen
En este artículo se describe el rendimiento académico del estudiantado de la región metropolitana y zonas alejadas, en la prueba nacional de matemática correspondiente a la conclusión de la educación media, considerando la prueba específica, según la modalidad y tipo de colegio: diurno (diurno científico, diurno humanístico), nocturno, técnico o centros integrados para la educación de jóvenes y adultos (CINDEA). El objetivo principal es describir el rendimiento académico estudiantil en las pruebas nacionales de bachillerato de matemática, correspondientes al año 2013, según las regiones educativas. Para el análisis de dicha información se utilizó como fuente primaria el Informe Nacional de Bachillerato, emitido por el Ministerio de Educación Pública para las pruebas estandarizadas del 2013. Una de las conclusiones, a partir de este estudio, es la necesidad de llevar a cabo un análisis histórico del rendimiento de las instituciones educativas que han obtenido, en los últimos años, los promedios más altos y más bajos en las pruebas de bachillerato de matemática, para poder profundizar en las causas de dichos rendimientos.
\end{abstract}

Palabras claves: Rendimiento académico, bachillerato, matemática.

\begin{abstract}
This article describes the academic performance of students from urban and distant areas in the national mathematics test corresponding to the completion of secondary education, considering the specific test and according to the different types of schools: daytime (daytime scientific, daytime humanistic), nighttime, technical or integrated centers for education of young people and adults (CINDEA, in its Spanish acronym). The main objective is to describe the students academic performance in the national
\end{abstract}


mathematics test issued to complete high-school level, for the year 2013 and according to the country educational areas. For the analysis of such information, the main source used was the High-School Education National Report, issued by the Ministry of Public Education for 2013 standardized tests. One of the conclusions from this study is the need to carry out a historical analysis of the performance of educational institutions which have recently obtained the highest and lowest average grades in the high-school diploma tests, in order to be able to delve into the causes of those performances.

Keywords: Academic performance; High school test; Mathematics.

El objetivo de este artículo es describir el rendimiento académico en las pruebas nacionales de bachillerato de matemática correspondientes al año 2013, aplicadas en las modalidades técnica y académica (diurna y nocturna) según las regiones educativas. Esto, por cuanto en ese año se inicia la implementación de un plan de transición de la reforma educativa en matemática, el cual consistió en trabajar con los programas de estudio de la educación general básica, en esta disciplina, aprobados desde 2005, enfatizando en el enfoque de resolución de problemas. También, a partir de ese año, la prueba nacional de bachillerato en matemática inicia un proceso de modificación paulatino, hasta alcanzar una prueba elaborada según el enfoque, contenidos, habilidades y metodología de los nuevos programas, proyectado para 2016.

Esta investigación se basó en el Informe Nacional de Bachillerato de la Educación Formal, del año 2013, emitido por el Ministerio de Educación Pública. A partir de los datos e información suministrada en dicho informe, se estableció un análisis específico para la prueba nacional de bachillerato en matemática, que consideró colegios académicos (diurnos y nocturnos) y técnicos. Asimismo, se consultó el tipo de colegio, público o privado, para ofrecer un panorama general respecto a la situación que se presentó en 2013 según esta variable.

La información que se ofrece a lo largo del artículo pretende ser un punto de partida para estudios y análisis posteriores, respecto al desarrollo y resultados de las pruebas nacionales en matemática a partir de la implementación de los nuevos programas de estudio.

\section{Panorama general de la educación media costarricense}

El Ministerio de Educación Pública, en Costa Rica, tiene a cargo la gestión administrativa, financiera y académica de la educación costarricense. Los servicios educativos de educación formal son instituciones que ofrecen opciones de educación a diferentes sectores, entre ellas se encuentran, en el nivel de educación media:

- Colegios diurnos

- Colegios nocturnos

- Colegios diurnos científicos y humanísticos

- Colegios técnicos

- Centros integrados para la educación de jóvenes y adultos (CINDEA)

- Colegios nocturnos nacionales virtuales Marco Tulio Salazar (CNVMTS)

Los colegios diurnos y nocturnos son instituciones que atienden el III Ciclo y la Educación Diversificada Académica a nivel nacional. En lo que respecta a los colegios diurnos científicos o humanísticos, es una modalidad diurna, regida a partir de proyectos de ley, en los cuales sus estudiantes ingresan a partir del noveno año y deben cumplir ciertos requisitos, particularmente 
de rendimiento académico. En este tipo de colegios, se enfatiza en áreas del conocimiento científico o humanístico con mayor profundidad que en el resto de colegios diurnos.

Los centros integrados (CINDEA) constituyen una oferta educativa integral para poblaciones de zonas rurales, urbanas y para el sistema penitenciario, dirigida a jóvenes-adultos y adultos que pretende beneficiar a esta población en áreas académicas, al desarrollo personal y social productivo, de forma que permita ofrecer mayores oportunidades educativas a través de un sistema flexible para el ingreso y avance de estudiantes.

Los colegios nacionales virtuales Marco Tulio Salazar son una alternativa académica virtual, que tiene como misión brindar oportunidades educativas a poblaciones sensibles entre los 15 y 18 años, como madres adolescentes, egresados y egresadas de tercer ciclo de secundaria, estudiantes que se han rezagado o que nunca se incorporaron al sistema educativo regular o que, por circunstancias especiales como económicas, laborales, geográficas, no pudieron acceder al sistema educativo tradicional.

\section{Las pruebas nacionales estandarizadas}

Las pruebas nacionales estandarizadas de bachillerato se vienen realizando en forma consecutiva desde finales de la década de los ochenta, para las disciplinas básicas: estudios sociales y cívica, ciencias (química, física o biología), matemática, inglés y español; su propósito ha sido valorar el nivel de apropiación de conocimientos básicos de la población estudiantil costarricense (Rojas, 1992; Pacheco, 1999). En el caso de la prueba de matemática, consiste en 60 preguntas de selección única correspondientes al temario oficial establecido por el MEP; la calificación final de bachillerato en cada asignatura está dada en un $60 \%$ por los resultados de la prueba y en un $40 \%$ por la media aritmética de las calificaciones de los dos o tres últimos años en las materias básicas (matemáticas, español, estudios sociales y cívica, una ciencia y una lengua extranjera), también conocida como nota de presentación. Esto se establece en el artículo 113 del Reglamento de Evaluación de los Aprendizajes del MEP.

Para la conclusión del sistema de educación media y poder optar por el ingreso a la universidad, es necesario obtener una calificación final de bachillerato igual o superior a 70 por ciento, en todas las asignaturas que componen el bloque de materias básicas (APSE, 2009).

El ente responsable de organizar, desarrollar y ejecutar la evaluación del sistema educativo ha sido la División de Control de Calidad y Macroevaluación del Sistema Educativo, desde el año 1998; anteriormente estaba a cargo del Centro Nacional de Evaluación para la Educación (Ministerio de Educación Pública, 1999).

\section{Prueba nacional de bachillerato en matemática}

La prueba nacional de bachillerato en la asignatura de matemática, al igual que las otras pruebas estandarizadas, ha generado expectativa año con año, respecto al nivel de destrezas matemática con las cuales el estudiantado de educación media finaliza sus estudios (Chaves, 2010).

Esta prueba estandarizada es aplicada en todas las regiones educativas del país. En este sentido, el Ministerio de Educación Pública considera 27 regiones entre el área metropolitana y las zonas alejadas. En el área metropolitana están las regiones de Alajuela, Heredia, Cartago, Desamparados, San José Central, San José Norte y San José Oeste; las zonas alejadas abarcan las regiones de Occidente, Limón, Pérez Zeledón, Los Santos, Aguirre, Santa Cruz, Guápiles, 
Puriscal, Turrialba, Puntarenas, San Carlos, Liberia, Nicoya, Cañas, Sarapiquí, Coto, Grande Térraba, Peninsular, Zona Norte-Norte y Sulá.

A partir de 1995, el Ministerio de Educación Pública realiza esfuerzos importantes para la divulgación y retroalimentación de la información producida por los resultados en las pruebas nacionales estandarizadas. De esta forma, se emiten anualmente informes de tipo ejecutivo, regional, para cada centro educativo, entre otros.

Según Esquivel (1996), respecto a la divulgación y retroalimentación que se ha dado a los resultados obtenidos en la pruebas de bachillerato por parte del Ministerio de Educación Pública, estos no han permitido producir materiales didácticos dirigidos a subsanar las deficiencias señaladas en los resultados, ni tampoco se ha suministrado dicha información a instituciones formadoras de docentes o aquellas encargadas de ofrecer capacitación a docentes en servicio.

Recientemente, a partir de una investigación realizada en la Universidad Nacional en el área específica de la enseñanza de la estadística, se obtuvieron las siguientes conclusiones:

- El profesorado de secundaria enfatiza en los contenidos que se indican en el temario de las pruebas, y el trabajo en clase se concentra en la resolución mecánica de ejercicios de similar dificultad.

- La evaluación sumativa que se realiza en el aula simula en gran medida a la prueba estandarizada.

- Existen, a disposición del estudiantado y docentes, libros de texto que están en función de las pruebas nacionales.

- Se ha enfatizado en el uso de la calculadora como estrategia para la resolución de ítems de selección única, sin dominio del concepto y procedimiento matemático de base (Chaves, 2010).

\section{Metodología}

La información utilizada para este artículo se obtuvo del Informe Nacional Bachillerato de la Educación Formal, del año 2013, emitido por el Ministerio de Educación Pública. Los datos utilizados corresponden específicamente a los resultados en la prueba, sin considerar la nota de presentación.

La estructura del análisis considera indicadores como: resultados en la prueba nacional de bachillerato según la región educativa, tipo de prueba según la modalidad diurno, nocturno o técnico, y tipo de colegio público o privado. El análisis efectuado por región se enfoca en los cinco mejores promedios o los cinco promedios más bajos obtenidos por institución educativa.

La cantidad de estudiantes que aplicaron la prueba de bachillerato en matemática del 2013, en el área metropolitana fue de aproximadamente 18543 y en zonas alejadas de 17587. Asimismo, la cantidad de estudiantes por modalidad de institución educativa fue de 22651 en colegios diurnos, 6992 en nocturnos y 6487 en técnicos.

En cuanto a los resultados en la prueba, en términos generales, muestran un promedio de nota en el examen de 61,45. El rendimiento académico, tal y como se muestra en la tabla 1, es mayor en 
ISSN Electrónico: 2215-3470

DOI: http://dx.doi.org/10.15359/ru.30-1.5
UNICIENCIA Vol. 30, No. 1, pp. 85-97. Enero-Junio, 2016. URL: www.revistas.una.ac.cr/uniciencia Email: revistauniciencia@una.cr

Tabla 1

Promoción en la prueba de bachillerato en matemática, por modalidad a nivel nacional, 2013

\begin{tabular}{lccc}
\hline Modalidad & $\mathrm{N}^{\circ}$ de colegios & $\mathrm{N}^{\circ}$ de estudiantes & Promedio de nota de examen \\
\hline Diurnos & 641 & 22651 & 63,94 \\
\hline Técnicos & 96 & 9487 & 58,11 \\
\hline Nocturnos & 258 & 6992 & 56,49 \\
\hline Nacional & 995 & 36130 & 61,45 \\
\hline
\end{tabular}

Nota: Ministerio de Educación Pública (2014, p. 20).

los colegios académicos diurnos, con un promedio de nota de examen de 63,94; y el rendimiento más bajo lo presentan los colegios nocturnos con un promedio de nota de examen de un 56,49.

A continuación se detalla la información analizada respecto a los resultados en la prueba de bachillerato del 2013.

\section{Distribución de la cantidad de ítems por objetivo o habilidad}

Las áreas que fueron evaluadas en la prueba nacional de bachillerato del 2013 correspondieron a las establecidas en el Programa de Matemática del 2005, a saber: álgebra; funciones; función exponencial y logarítmica; geometría y trigonometría.

Como lo muestra la tabla 2, el área y las habilidades más evaluadas en la prueba de bachillerato

Tabla 2

Distribución de ítems en la prueba de bachillerato por

área de conocimiento, 2013

\begin{tabular}{lc}
\hline \multicolumn{1}{c}{ Área } & Número de ítems \\
\hline Álgebra & 7 \\
\hline Funciones & 26 \\
\hline Función exponencial y logarítmica & 10 \\
\hline Geometría & 14 \\
\hline Trigonometría & 3 \\
\hline Total & 60 \\
\hline
\end{tabular}

Nota: Elaboración propia del estudio.

del 2013 corresponden al área de funciones, con un total de 26 ítems. El área menos evaluada fue trigonometría con un total de 3 ítems. 
UNICIENCIA Vol. 30, No. 1, pp. 85-97. Enero-Junio, 2016.

ISSN Electrónico: 2215-3470

URL: www.revistas.una.ac.cr/uniciencia

Email: revistauniciencia@una.cr

Cabe destacar que la prueba nacional hace énfasis en los conocimientos sobre el análisis de gráficas de funciones y resolución de problemas a partir de la aplicación del álgebra.

\section{Resultados}

\section{Rendimiento de los estudiantes por región educativa y tipo de prueba}

El Ministerio de Educación Pública, cada año, aplica pruebas de bachillerato diferenciadas, según la modalidad (diurna, nocturna o técnica) y la región, sea área metropolitana o zonas alejadas. La prueba M-21 corresponde a los colegios nocturnos y la prueba M-31 se aplica en los técnicos. La prueba M-11 la realizan los colegios diurnos del área metropolitana, específicamente las regiones de San José Norte, San José Oeste, San José Central, Desamparados, Heredia, Cartago y Alajuela. Y la prueba M-12 se aplica a colegios diurnos de veinte regiones educativas fuera del área metropolitana. Para el análisis de estas pruebas se establecen tres categorías de rendimiento: a) aceptable, que corresponde a una calificación igual o superior a 70 o contar con 42 o más respuestas correctas en la prueba; b) moderada, son calificaciones mayores o iguales a 50 y menores a 70 o de 30 a 41 respuestas correctas en la prueba; y, c) deficiente, que son calificaciones inferiores a 50 o 29 o menos respuestas correctas en la prueba.

En ninguno de los colegios diurnos del área metropolitana (prueba M-11), se supera el $40 \%$ en la categoría de aceptable; y aquellas que obtuvieron las mejores calificaciones, de 36,5\% y $32,4 \%$ corresponden respectivamente a San José Norte y San José Oeste, es decir, se ubican en la capital. Mientras que las regiones que presentan mayores porcentajes en la categoría de deficiente y que superan el 50\%, corresponden a Alajuela, San José Central y Desamparados, esta última con el porcentaje más alto.

En los colegios diurnos de las zonas alejadas donde se aplicó la prueba M-12, la situación que se presenta es preocupante en el sentido de que los porcentajes de estudiantes que se ubican en la categoría deficiente oscilan entre un $37,9 \%$ y un $90 \%$, es decir, existe una gran variabilidad; en particular, las regiones de Sulá y Peninsular muestran el alarmante porcentaje de $90 \%$ y 89,5\%, respectivamente. Casos críticos como la zona de Peninsular no presenta ningún estudiante aprobado en la prueba de bachillerato, es decir, el porcentaje de la categoría de aceptable es nulo. Por su parte, las zonas que presentan mayores porcentajes en la categoría de aceptable, son Santa Cruz y Limón; no obstante, las calificaciones de aceptable, en esta población estudiantil, no alcanzan el 40\%.

Con respecto a la prueba M-21, aplicada a los colegios nocturnos del país, se observa que, con excepción de Limón, el resto de las zonas alejadas presentan porcentajes entre el 40,1\% y $93,8 \%$ en la categoría de deficiente. Se presentan casos críticos como la zona de Los Santos y Sarapiquí, en las que ningún estudiante aprobó la prueba de bachillerato. También zonas como San José Central, San José Norte, Liberia y Cañas presentan porcentajes inferiores al 1\% en la categoría de aceptable. Por el contrario, en forma positiva sobresale la zona de Limón, la cual tiene un porcentaje de $81,7 \%$ en la categoría de aceptable.

En cuanto a la prueba M-31 aplicada a la población de colegios técnicos, el porcentaje de examinados en la categoría de deficiente oscila entre 8,9\% y 91,1\%, como puede notarse la variabilidad de los datos es alta, es la región de Sulá la que presenta el porcentaje más bajo $(8,9 \%)$; esta situación se traduce en que ningún estudiante se ubicó en la categoría aceptable en dicha región, es decir, nadie aprobó la prueba nacional con nota superior o igual a 70. Similar 
situación ocurrió en la zona de Santa Cruz, en la cual ningún estudiante aprobó la prueba nacional y el $91,1 \%$ se ubicó en la categoría de deficiente. Cañas es la zona que presenta el mayor porcentaje en la categoría de aceptable, con un $40 \%$.

\section{Rendimiento y nivel de desempeño institucional, por región, modalidad y tipo de colegio}

En este apartado se describe el rendimiento académico del estudiantado evaluado en la prueba de bachillerato del 2013, según área metropolitana o zonas alejadas. Se hace énfasis en el promedio obtenido por las cinco mejores instituciones y las cinco instituciones con promedios más bajos.

En lo que respecta al área metropolitana, como se mencionó con anterioridad, está conformada por las regiones educativas de Alajuela, Cartago, Heredia, San José Central, San José Norte y San José Oeste.

En Alajuela, la institución con el mejor promedio fue el Colegio Científico con un promedio de 97,4 y una desviación estándar de 2,4; es decir, la mayoría de sus estudiantes obtuvieron notas superiores a 90 en la prueba. El segundo, tercer y cuarto colegio con mejores promedios fueron de tipo privado. Cabe destacar, que el quinto mejor promedio lo presentó el CAI San Rafael y Adulto Mayor, centros de atención institucional, en los cuales cuatro estudiantes que aplicaron la prueba obtuvieron una nota mayor o igual a 70 .

En contraposición, los cinco colegios que presentan los promedios más bajos de la prueba en la región de Alajuela, son públicos y la mayoría de modalidad nocturna virtual.

En la región educativa de Cartago, cuatro de los cinco colegios con los mejores promedios en la región son públicos, a saber, el Colegio Científico de Cartago (mejor promedio, con notas superiores a 90), el Liceo Experimental Bilingüe José Figueres, el Colegio Técnico Profesional COVAO y el Colegio San Luis Gonzaga. El colegio que ocupa el quinto lugar entre los mejores promedios en la prueba es el Bilingüe Sonnysa, de carácter privado. Por otro lado, tres de los colegios con promedios más bajos (con notas inferiores a 35), al igual que en Alajuela, son colegios nocturnos virtuales.

En la región educativa de Desamparados, el único colegio dentro de los cinco mejores que obtuvo un promedio mayor a 70, es el colegio privado San Miguel Arcángel. Además, de manera recurrente, tres de los cinco colegios con promedios más bajos en esta zona, corresponden a colegios nocturnos virtuales.

En Heredia, destaca el Colegio Humanístico Costarricense, como único colegio público dentro de los mejores cinco promedios. El resto de colegios ubicados entre los primeros promedios, son colegios privados o subvencionados. En cuanto a la modalidad de dichos colegios, todos son diurnos. Los colegios que obtuvieron las más bajas calificaciones en la prueba son colegios nocturnos virtuales y un centro de atención integral de niños y niñas.

En la región educativa San José Central, los mejores cinco promedios corresponden a instituciones privadas y tres de los cinco colegios con promedios más bajos son centros nocturnos virtuales. Sin embargo, llama la atención el caso del colegio Dr. José Ma. Castro Madriz, en el cual, con una población de 112 estudiantes que presentaron la prueba, ninguno la aprobó.

En San José Norte, cuatro de los cinco mejores promedios en la prueba son instituciones privadas, no obstante, destaca el colegio científico de San Pedro, de carácter público, con el mejor promedio. Por otra parte, en cuanto a las instituciones con rendimientos bajos, el Colegio de Purral, de modalidad diurna, con tan solo 7 estudiantes, presenta el promedio más bajo. 
UNICIENCIA Vol. 30, No. 1, pp. 85-97. Enero-Junio, 2016.

En la región educativa San José Oeste, los cinco mejores promedios corresponden a colegios privados. De la misma manera, en cuanto a los colegios con menor rendimiento, tres de las cinco instituciones con promedios más bajos son de modalidad nocturna, y dos corresponden a colegios nacionales virtuales.

En lo que respecta a zonas alejadas, son 21 regiones educativas las que componen este sector. A continuación se detalla el rendimiento académico por región, tipo de colegio y modalidad de las instituciones educativas en esta zona.

En la tabla 3 se muestran los cinco mejores promedios de la región de Aguirre, en donde destaca como mejor promedio el Colegio Nocturno de Jacó, con un 79,6 y una desviación

Tabla 3

Rendimiento académico de la región de Aguirre, por tipo de colegio y modalidad, 2013

\begin{tabular}{lccccc}
\hline Liceo & N & Promedio & $\begin{array}{l}\text { Desviación } \\
\text { estándar }\end{array}$ & $\begin{array}{l}\text { Tipo de } \\
\text { colegio }\end{array}$ & Modalidad \\
\hline Colegio Nocturno de Jacó & 14 & 79,6 & 8,3 & Público & Nocturno \\
\hline Bilingüe Inmaculada Jacó & 5 & 73,3 & 10,3 & Privado & Diurno \\
\hline Las nubes School & 4 & 69,6 & 29,3 & Privado & Diurno \\
\hline C.T.P. de Matapalo & 17 & 69,5 & 12,0 & Público & Técnico \\
\hline Los delfines & 10 & 67,5 & 3,6 & Privado & Diurno \\
\hline
\end{tabular}

Nota: Ministerio de Educación Pública (2014, p. 411).

estándar de 8,3, lo que supone que la mayoría de estudiantes aprobó el examen de bachillerato. Posicionado en el tercer lugar está el CTP de Matapalo, con un promedio de 69,5 y una desviación estándar de 12,0; esto sugiere que más de la mitad de los estudiantes obtuvo notas mayores o iguales a 70. Por otra parte, solo dos colegios tienen un promedio superior a 70.

Al analizar los promedios más bajos, es preocupante que nueve colegios de los veinte que constituyen la región de Aguirre, entre diurnos, nocturnos y técnicos presentaran 100\% de reprobación.

Tabla 4

Rendimiento académico de la región de Cañas, por tipo de colegio y modalidad, 2013

\begin{tabular}{lccccc}
\hline Liceo & N & Promedio & $\begin{array}{c}\text { Desviación } \\
\text { estándar }\end{array}$ & $\begin{array}{l}\text { Tipo de } \\
\text { colegio }\end{array}$ & Modalidad \\
\hline San Daniel Comboni & 12 & 83,8 & 7,5 & Privado & Diurno \\
\hline San Jorge & 32 & 64,4 & 14,4 & Privado & Diurno \\
\hline Eulogio López Obando & 24 & 64,1 & 17,2 & Privado & Diurno \\
\hline Liceo Maurilio Alvarado Vargas & 99 & 49,4 & 18,2 & Público & Diurno \\
\hline $\begin{array}{l}\text { Liceo Experimental Bilingüe Nuevo } \\
\text { Arenal }\end{array}$ & 24 & 48,9 & 8,9 & Público & Diurno \\
\hline
\end{tabular}

Nota: Ministerio de Educación Pública (2014, pp. 413). 
En la región educativa de Cañas, como se muestra en la tabla 4, el mejor promedio es un colegio privado, con modalidad diurna, el cual presenta un promedio de 83,8 . No obstante, preocupa el hecho de que únicamente esta institución presente un promedio superior a 70, y que dos de las instituciones con mejores promedios de la zona presenten promedios de 49,4 y 48,9.

En cuanto a los promedios más bajos de la región educativa de Cañas, tres de los cinco colegios con promedios más bajos son nocturnos y dos de ellos virtuales.

$\mathrm{Al}$ analizar la región de Coto, destaca el Colegio Humanístico de Coto como la institución educativa con el mejor promedio, con un 87,2 y una desviación estándar de 10,2. A excepción del Humanístico, las otras instituciones ubicadas entre las cinco mejores no alcanzaron un promedio mayor a 70 en la prueba. En esta región, las instituciones que presentan los promedios más bajos, menores a 30, obtuvieron un $100 \%$ de reprobación; tres de dichas instituciones son diurnas, una técnica y una nocturna, todas públicas.

En la región Grande Térraba, entre los mejores promedios se ubica el colegio privado PINDECO y cuatro liceos públicos, entre ellos, el Liceo Rural Changuena y el Colegio Nocturno CNVMTS Sede Escuela Coronado. No obstante, preocupa el hecho de que en el 55\% de las instituciones educativas de la región, los estudiantes obtuvieron notas menores a 70.

En la zona educativa de Guápiles, por otra parte, los dos mejores promedios corresponden a colegios privados y en ambas instituciones la mayoría de los estudiantes obtuvieron notas mayores o iguales a 70 en la prueba. El tercer mejor promedio es público y lo constituye el Liceo Experimental Bilingüe de Pococí. Al igual que en la región Grande Térraba, un alto porcentaje, es decir, en un 58\% de las instituciones, sus estudiantes no alcanzaron una nota igual o superior a 70 en la prueba. Y las instituciones con promedios más bajos corresponden a colegios nocturnos.

Tabla 5

Rendimiento académico de la región de Liberia, por tipo de colegio y modalidad, 2013

\begin{tabular}{llllll}
\hline Liceo & N & Promedio & $\begin{array}{l}\text { Desviación } \\
\text { estándar }\end{array}$ & $\begin{array}{l}\text { Tipo de co- } \\
\text { legio }\end{array}$ & Modalidad \\
\hline Colegio Científico de Liberia & 9 & 93,5 & 2,6 & Público & Diurno \\
\hline Escuelas Intern. Cristianas & 18 & 86,9 & 6,3 & Privado & Diurno \\
\hline Teocali & 21 & 78,9 & 11,9 & Privado & Diurno \\
\hline Colegio Intern. De Guanacaste & 6 & 63,6 & 13,3 & Privado & Diurno \\
\hline Santa Ana & 76 & 60,5 & 16,7 & Privado & Diurno \\
\hline
\end{tabular}

Nota: Ministerio de Educación Pública (2014, p. 418).

En Liberia, como lo muestra el tabla 5, a excepción del Colegio Científico de Liberia, los colegios que ocupan las cinco primeras posiciones en los promedios de la prueba son privados. Sin embargo, dos de ellos, a pesar de estar entre los colegios con mejores promedios, no alcanzan la nota mínima de 70 en su promedio.

Los colegios que obtuvieron los promedios más bajos en la región educativa de Liberia, muestran un 100\% de reprobación, y en casos como el Liceo Rural Piedras Azules y el Liceo 
UNICIENCIA Vol. 30, No. 1, pp. 85-97. Enero-Junio, 2016.

ISSN Electrónico: 2215-3470

URL: www.revistas.una.ac.cr/uniciencia

DOI: http://dx.doi.org/10.15359/ru.30-1.5

Email: revistauniciencia@una.cr

Tabla 6

Rendimiento académico de la región de Limón, por tipo de colegio y modalidad, 2013

\begin{tabular}{llllll}
\hline Liceo & N & Promedio & $\begin{array}{l}\text { Desviación } \\
\text { estándar }\end{array}$ & $\begin{array}{l}\text { Tipo de } \\
\text { colegio }\end{array}$ & Modalidad \\
\hline $\begin{array}{l}\text { Colegio Científico del At- } \\
\text { lántico }\end{array}$ & 12 & 96,5 & 3,1 & Público & Diurno \\
\hline Gilande & 5 & 90,7 & 1,5 & Privado & Diurno \\
\hline CINDEA Limón Central & 67 & 84,9 & 12,5 & Público & Nocturno \\
\hline CINDEA 28 Millas Palacios & 5 & 84,3 & 2,5 & Público & Nocturno \\
\hline CINDEA Limón Sandoval & 3 & 83,9 & 1,9 & Público & Nocturno \\
\hline
\end{tabular}

Nota: Ministerio de Educación Pública (2014, p. 419).

Juntas de Caoba el 100\% de estudiantes obtuvo notas inferiores a 50.

En contraste con la realidad planteada en la región de Liberia, los cinco mejores promedios en la región de Limón son colegios nocturnos y centros integrales de educación para adultos, tal y como se muestra en la tabla 6.

Sin embargo, no todos los colegios públicos presentan un buen rendimiento en esta región de Limón, de hecho los cinco colegios que obtuvieron los promedios más bajos son públicos, y ninguno de los estudiantes en dichas instituciones aprobó la prueba.

En la región educativa de los Santos, la situación presentada en esta prueba llama la atención, en tanto solo el Liceo Rural Santa Cruz obtuvo un promedio mayor a 70. Los restantes colegios no superan un 55 de promedio en la prueba.

La situación en la región de Nicoya es similar a la presentada en Limón, donde tres de los mejores promedios de la región de Nicoya son nocturnos, dos centros integrales de educación para adultos (CINDEA Nicoya Copal y Nicoya Santa Ana) y un centro nocturno virtual (Sede CTP La Mansión), todos públicos. No obstante, el mejor promedio corresponde a un colegio privado, el Instituto Pedagógico EUPI. Cabe mencionar que en esta región solo dos instituciones tienen notas superiores a 70 .

Además, las instituciones de esta región, con promedios más bajos presentan una reprobación del $100 \%$, y sus promedios no superan el 40 .

En la región de Occidente, los mejores promedios corresponden a instituciones públicas, diurnas. El primer promedio lo tiene el Colegio Científico de San Ramón, con un promedio de 93,7 y una desviación estándar de 7. Por otra parte, los promedios más bajos corresponden a colegios nocturnos o diurnos (CINDEA Y TV) con calificaciones que no superan el 40.

En la región Peninsular, es preocupante que, en toda la región, el único colegio con un promedio superior a 70 corresponde al CINDEA Jicaral-Cóbano. El resto de instituciones presenta una nota inferior a 45 en el promedio de la prueba.

La región de Pérez Zeledón presenta una situación un poco mejor; sin embargo, solo dos colegios muestran promedios superiores a 70, el Colegio Científico de Pérez Zeledón, público y el Liceo del Valle, privado. Se destaca el número de estudiantes que aplicaron la prueba en el Liceo Unesco, 235, y que a pesar de ello, es el quinto mejor promedio. Además, cabe mencionar que los 
cinco mejores promedios de la región son de modalidad diurno. Asimismo, los cinco promedios más bajos en Pérez Zeledón corresponden a instituciones públicas con notas inferiores a 40.

La región educativa de Puntarenas presenta, en los primeros cinco lugares, notas superiores a 75 , donde cuatro de los cinco mejores promedios corresponden a colegios públicos, uno de ellos en modalidad nocturna y el resto son diurnos. Destaca el Colegio Científico de Puntarenas con el mejor promedio de la región, un 90,7 con una desviación estándar de 7,7. En relación con los cinco colegios con promedios más bajos en Puntarenas, son nocturnos, públicos y su promedio es inferior a 31.

En la región educativa de Puriscal, únicamente el Liceo Coronel Manuel Arguello, público y diurno, supera un 70 de promedio, el resto tiene promedios realmente bajos que llegan a 17,2 con una desviación estándar de 13,9. En toda la región, solo dos instituciones son de carácter privado, una de ellas se ubica entre los mejores promedios y otra entre las peores.

Para la región de San Carlos solo tres promedios de la región superan el 70, dos de ellos privados y el otro corresponde al Colegio Científico de San Carlos, el cual tiene un promedio de 92,3 y una desviación estándar de 8,5. Preocupan los promedios de las instituciones con menor rendimiento, pues las cinco instituciones con promedios más bajos presentan promedios inferiores a 35 . Ninguno de los estudiantes en dichos colegios aprobó el examen, y obtuvieron notas inferiores a 50.

Para la región educativa de Santa Cruz, los promedios no son muy altos, apenas alcanzan un 75,8; destaca el hecho de que las dos instituciones con mejores promedios son colegios nocturnos, uno virtual y un CINDEA. Seguidos de dos instituciones privadas.

En esta región, las instituciones con menores promedios en la prueba nacional corresponden a colegios diurnos y técnicos, y no alcanzan un promedio superior a 35.

En Sarapiquí es preocupante el rendimiento general de la región, pues ningún colegio en la región, público o privado, supera un promedio de 62. Además, 16 de 23 colegios presentaron un 100\% de reprobación en el examen.

En la región de Sulá, los dos mejores promedios son instituciones nocturnas, además

Tabla 7

Rendimiento académico de la región de Sulá, por tipo de colegio y modalidad, 2013

\begin{tabular}{lccclc}
\hline \multicolumn{1}{c}{ Liceo } & N & Promedio & $\begin{array}{c}\text { Desviación } \\
\text { estándar }\end{array}$ & $\begin{array}{l}\text { Tipo de } \\
\text { colegio }\end{array}$ & Modalidad \\
\hline Liceo Rural Katsi & 10 & 20,5 & 7,3 & Público & Diurno \\
\hline Liceo Rural San Vicente & 6 & 21,9 & 2,5 & Público & Diurno \\
\hline Liceo Rural Alto Cohen & 4 & 22,5 & 7,9 & Público & Diurno \\
\hline Liceo Rural Shiroles & 3 & 24,4 & 5,1 & Público & Diurno \\
\hline Liceo Rural Coroma & 10 & 25,3 & 3,7 & Público & Diurno \\
\hline
\end{tabular}

Nota: Ministerio de Educación Pública (2014, p. 430).

corresponden a CINDEA. Se destaca el CINDEA Bribri-Cahuita con un 100\% de estudiantes con notas superiores a 70 .

Los cinco colegios con el rendimiento más bajo en Sulá son diurnos y públicos, y el 100\% de 
UNICIENCIA Vol. 30, No. 1, pp. 85-97. Enero-Junio, 2016.

Email: revistauniciencia@una.cr

sus estudiantes no alcanzaron notas superiores a 50, como se observa en la tabla 7.

En la región educativa de Turrialba, únicamente dos colegios presentan un promedio superior a 70, ambos de carácter privado. Además, cuatro de los cinco colegios con más bajo rendimiento corresponden a colegios nocturnos virtuales.

Tabla 8

Rendimiento académico de la región de Norte-Norte, por tipo de colegio y modalidad,2013

\begin{tabular}{llllll}
\hline \multicolumn{1}{c}{ LICEO } & N & Promedio & $\begin{array}{c}\text { Desviación } \\
\text { estándar }\end{array}$ & $\begin{array}{c}\text { Tipo de } \\
\text { Colegio }\end{array}$ & Modalidad \\
\hline Liceo Colonia Villa Nueva & 10 & 55,8 & 19,4 & Público & Diurno \\
\hline Liceo de San José (Upala) & 32 & 55,1 & 17,0 & Público & Diurno \\
\hline Liceo Cuatro Bocas & 6 & 55,0 & 25,5 & Público & Diurno \\
\hline Liceo Rural Veracruz & 6 & 53,1 & 11,6 & Público & Diurno \\
\hline Liceo Katira & 30 & 47,8 & 11,9 & Público & Diurno \\
\hline
\end{tabular}

Nota: Ministerio de Educación Pública (2014, p. 430).

Como se observa en la tabla 8 , la zona Norte-Norte es una región con una problemática grave en cuanto al rendimiento en matemática, ya que ninguna institución alcanza ni siquiera un 60 de promedio. El promedio más alto es de un 55,8 y los colegios con rendimientos más bajos son nocturnos y CINDEA.

\section{Conclusiones}

A partir del análisis efectuado se pueden evidenciar las siguientes conclusiones:

1. Las regiones educativas del área metropolitana presentan, en general, un mejor rendimiento en la prueba nacional de bachillerato del 2013, en comparación con las zonas alejadas.

2. En algunas regiones educativas del país, como Pérez Zeledón, Limón, Liberia, Coto, los colegios científicos y humanísticos surgen como una opción de calidad de carácter público. Estas instituciones, como se evidencia a lo largo del artículo, presentan los mejores promedios en las regiones educativas donde se ubican.

3. Existen instituciones de zonas alejadas, como el Colegio nocturno de Jacó y CINDEA en la región de Limón, que destacan por su alto rendimiento, a pesar de las condiciones socioeconómicas de su población y de las características propias de la región.

4. La modalidad de colegio nocturno virtual en la mayoría de las regiones educativas del país presenta muy bajos rendimientos, aspecto que debe ser atendido y supervisado por el Ministerio de Educación Pública.

5. En regiones como Heredia, San José Norte, San José Oeste y San José Central, a excepción de los colegios científicos o humanísticos, las instituciones que presentan mejor rendimiento en la prueba de bachillerato son de tipo privado. 
ISSN Electrónico: 2215-3470

DOI: http://dx.doi.org/10.15359/ru.30-1.5
UNICIENCIA Vol. 30, No. 1, pp. 85-97. Enero-Junio, 2016.

URL: www.revistas.una.ac.cr/uniciencia Email: revistauniciencia@una.cr

6. En esta prueba estandarizada del 2013, regiones educativas como Sarapiquí y Zona Norte- Norte, no presentaron instituciones con promedios superiores a 70. Y en el caso de regiones como Cañas, Coto, Zona de los Santos, Peninsular y Puriscal solo se registró una institución con promedio superior a 70.

\section{Referencias}

APSE. (2009). Reglamento de Evaluación de los Aprendizajes. En Leyes y Reglamentos Docentes. Asociación de Profesores de Segunda Enseñanza. Recuperado de http://www.apse.or.cr/WebApse/legdoc/leg03.htm.

Chaves, E. (2010). Las pruebas estandarizadas de matemáticas: Lo bueno y lo malo, opinión de docentes. Acta Latinoamericana de Matemática Educativa, 23, 257-265.

Esquivel, J. (1996). Medición de logros de aprendizaje y el empleo de los resultados en Costa Rica. En Seminario internacional de evaluación y estándares de la educación en América Latina. Realidades y desafíos. PREAL, Río de Janeiro, 4-5 de diciembre.

Ministerio de Educación Pública. (1999). Informe nacional de resultados de las pruebas nacionales de la educación formal 1998. Bachillerato. Dirección de Gestión y Evaluación de la Calidad. San José, Costa Rica.

Ministerio de Educación Pública. (2014). Informe nacional de resultados de las pruebas nacionales de la educación formal 2013. Bachillerato. Dirección de Gestión y Evaluación de la Calidad. San José, Costa Rica.

Pacheco, F. (1999). Evaluación y pruebas nacionales. En Política social y educación en Costa Rica (pp. 257- 277). San José, Costa Rica: UNICEF- Costa Rica.

Rojas, Y. (1992). Transformaciones recientes en la educación costarricense. En El Nuevo Rostro de Costa Rica (pp. 97 -121). Costa Rica: Cedal.

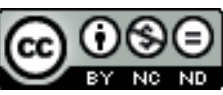

Rendimiento académico en la prueba estandarizada de bachillerato de matemática en colegios del área metropolitana y zonas alejadas de Costa Rica en 2013 (Mario Castillo-Sánchez y otros.) por Revista Uniciencia se encuentra bajo una Licencia CreativeCommons Atribución-NoComercial-SinDerivadas 3.0 Unported. 